\title{
The CC3 model: An iterative coupled cluster approach including connected triples
}

\author{
Henrik Koch, Ove Christiansen, and Poul Jørgensen \\ Department of Chemistry, Aarhus University, DK-8000 Aarhus C, Denmark \\ Alfredo M. Sanchez de Merás \\ Universidad de Valencia, Facultad de Ciencias Quimicas, Departamento de Quimica Fisica, \\ Doctor Moliner, 50, Burjasot (Valencia), Spain \\ Trygve Helgaker \\ Department of Chemistry, University of Oslo, P.O. Box 1033 Blindern, N-0315 Oslo, Norway
}

(Received 18 July 1995; accepted 11 September 1996)

\begin{abstract}
An alternative derivation of many-body perturbation theory (MBPT) has been given, where a coupled cluster parametrization is used for the wave function and the method of undetermined Lagrange multipliers is applied to set up a variational coupled cluster energy expression. In this variational formulation, the $n$ th-order amplitudes determine the energy to order $2 n+1$ and the $n$ th-order multipliers determine the energy to order $2 n+2$. We have developed an iterative approximate coupled cluster singles, doubles, and triples model CC 3 , where the triples amplitudes are correct through second order and the singles amplitudes are treated without approximations due to the unique role of singles as approximate orbital relaxation parameters. The compact energy expressions obtained from the variational formulation exhibit in a simple way the relationship between CC3, CCSDT-1a [Lee et al., J. Chem. Phys. 81, 5906 (1984)] CCSDT-1b models [Urban et al., J. Chem. Phys. 83, 4041 (1985)], and the CCSD(T) model [Raghavachari et al., Chem. Phys. Lett. 157, 479 (1989)]. Sample calculations of total energies are presented for the molecules $\mathrm{H}_{2} \mathrm{O}$, $\mathrm{C}_{2}, \mathrm{CO}$, and $\mathrm{C}_{2} \mathrm{H}_{4}$. Comparisons are made with full CCSDT, CCSDT-1a, CCSDT-1b, CCSD(T), and full configuration interaction (FCI) results. These calculations demonstrate that $\mathrm{CC} 3$ and $\operatorname{CCSD}(\mathrm{T})$ give total energies of a similar quality. If results obtained by $\mathrm{CC} 3$ and $\operatorname{CCSD}(\mathrm{T})$ differ significantly, neither method can be trusted. In contrast to $\operatorname{CCSD}(\mathrm{T})$, time-dependent response functions can be obtained for CC3. (c) 1997 American Institute of Physics.
\end{abstract}

[S0021-9606(97)01747-3]

\section{INTRODUCTION}

The coupled cluster $(\mathrm{CC})$ model was introduced in the early 1960s and has since gained increasing popularity. The coupled cluster model is size extensive and describes in an efficient way the dynamical correlation. Today the CC singles and doubles (CCSD) model is one of the most commonly used methods for obtaining an accurate correlated description of the electronic structure of single configuration dominated systems. ${ }^{1}$ The CCSD scale as $N^{6}$, where $N$ is the number of orbitals. Extensions of CCSD to a singles, doubles, and triples (CCSDT) model ${ }^{2,3}$ is desirable in order to obtain a more accurate solution and also because triples have shown some ability to recover static correlation contributions. CCSDT calculations scale as $N^{8}$ and triples amplitudes must be stored. It is therefore desirable to develop methods where triples are treated in an approximate fashion. Two strategies have been advocated, both of which may avoid the storage of triples amplitudes and reduce the computational effort to that of an $N^{7}$ algorithm. In the first approach, simplifications are introduced in the CCSDT amplitudes equations, and iterative methods are used to solve the amplitude equations. ${ }^{4-6}$ In the other and more popular approach, the lowest-order perturbation energy contributions from connected triples are added to the CCSD energy. ${ }^{6-9}$
The CCSD energy is correct through fourth order except for one fourth-order contribution from connected triples. This contribution is added in all perturbation energy corrected CCSD models. In the first of these CCSD $+T$ (CCSD), only the fourth-order energy contribution was added, but with CCSD cluster amplitudes replacing the doubles correlation coefficients. The connected triples give one fifth-order contribution involving singles amplitudes. If this contribution is added to the CCSD energy together with the connected triples fourth-order contribution, but with the CCSD single and double amplitudes replacing the single and double correlation coefficients, then the $\operatorname{CCSD}(\mathrm{T})$ model is obtained. ${ }^{8}$ This approach is the most widely used approximate triples model and is also observed to be the best behaved. However, the CCSD(T) model is unsatisfactory from a pure perturbational point of view, in the sense that we include two particular fifth-order triples contributions and disregard all others, with no apparent justification. The effect of considering all fifth-order contributions has been considered by Bartlett et al. ${ }^{10}$

The $\operatorname{CCSD}(\mathrm{T})$ and all noniterative perturbation corrected CCSD models are two-step procedures. First a CCSD calculation is carried out to determine the amplitudes and energy, next the effect of connected triples is taken into account by 
adding low-order perturbation triples energy terms that are absent in the CCSD model. Response functions cannot be obtained in a consistent manner from such two-step approaches. For example, the $\operatorname{CCSD}(\mathrm{T})$ model has a pole structure corresponding to that of the CCSD wave function. Therefore, excitation energies and frequency-dependent molecular properties cannot be obtained that are consistent with the $\operatorname{CCSD}(\mathrm{T})$ model. The $\operatorname{CCSD}(\mathrm{T})$ model can thus only be applied to ground state energies and static molecular properties. Response functions with a well-defined pole structure, i.e., with only one set of poles obtained at the level of approximation of the reference wave function, can only be obtained in models where the determination of parameters and energy can be viewed as a one-step approach. This is the case for the CC3 model and for the models where approximate triples amplitude equations are solved iteratively.

If the triples equation is approximated according to second-order perturbation theory, the cluster amplitude equations of the CCSDT-1b model of Urban et al. are obtained. ${ }^{6}$ The CCSDT-1b model is one of the many models proposed by Urban et al., where approximations are introduced in the CCSDT amplitude equations to arrive at models that scale as $N^{7}$. The CCSDT-1a model is obtained from CCSDT-1b by neglecting the singles in the connected triples contributions to the doubles equations. CCSDT-1a appears to be the most popular iterative approximation to CCSDT.

Perturbation analysis based on the Hartree-Fock energy shows that singles occur to second order in the wave function and to fourth order in the energy. They therefore appear to be much less important than the doubles, which occur to first order in the wave function and second order in the energy. However, if an external perturbation is applied to the system, the singles are first order in the external perturbation and zeroth order in the fluctuation potential. Singles are thus more important than suggested by the energy. The singles have the unique role of being approximate orbital relaxation parameters. We therefore require that the CC3 model introduces no approximations in the treatment of singles. Thus, the singles amplitudes are treated as zeroth order in the fluctuation potential. We further require $\mathrm{CC} 3$ to treat triples correct to second order. In this way we obtain an iterative approximate triples model that is well suited for describing molecular properties. In a separate paper we have derived the CC3 linear response function. This includes a pole and residue analysis to determine $\mathrm{CC} 3$ excitation energies and transition matrix elements. The $\mathrm{CC} 3$ linear response function has also been compared to the ones of the CCSDT-1a and CCSDT-1b models. The special treatment of singles in CC3 has been demonstrated to be extremely important in molecular property calculations, for example, single replacement dominated excitation energies are correct through third order in CC3 and only through second order in CCSDT-1a and CCSDT-1b. The use of the CCSDT-1 ground state wave function was first presented by Watts and Bartlett. Benchmark calculations have demonstrated that little or no improvement are obtained in CCSDT-1 for single replacement dominated excitation energies compared to CCSD while significant improvement is obtained in CC3. We refer to Ref. 11 for a detailed derivation and discussion of the properties of the CC3 linear response function.

To examine the structure of $\mathrm{CC} 3$ and other approximate triples models we present an alternative and simple derivation of many-body perturbation theory (MBPT). In this derivation, we use a coupled cluster parametrization of the exact wave function to ensure that only size-extensive contributions appear in the perturbation expansion. Perturbation theory based on a coupled cluster parametrization of the wave function has been considered by many authors; see Kucharski and Bartlett ${ }^{12}$ for a recent reference. We further introduce a variational coupled cluster energy expression. To understand how this is done recall that the CC total energy is calculated from amplitudes that are obtained by solving the amplitude equations. Thus the total energy is formally obtained from a constrained optimization. The CC energy can therefore be viewed as obtained from an unconstrained optimization of a variational energy expression where the cluster amplitude equations are added as constraints using the method of undetermined Lagrange multipliers. Clearly, this gives no simplification in the calculation of the coupled cluster total energy. However, when calculating perturbational approximations to the coupled cluster (exact) energy, the variational property of this CC Lagrangian becomes important, as the energy through order $2 n+1$ can be obtained from the $n$ th-order correction to the cluster amplitudes and Lagrange multipliers. In fact, the $n$ th-order Lagrange multipliers determine the energy to order $2 n+2 .{ }^{13}$ The use of the $2 n+1$ and $2 n+2$ rules leads to compact energy expressions. The coupled cluster parametrization ensures that only connected contributions are contained in the energy expansion.

The Lagrangian method is a completely general method for handling nonvariational energies. In the special case of coupled cluster theory with fixed orbitals, as in this paper, it becomes equivalent to the method of Arponen ${ }^{14}$ in the framework of the "normal $\exp (S)$ '" method. In the context of obtaining an efficient evaluation of coupled cluster energy gradient the derivative of the variational functional was obtained by Bartlett. ${ }^{15}$ The functional itself was first used in quantum chemistry by Helgaker and Jørgensen ${ }^{16}$ to derive coupled cluster energy derivatives. The full strength of a variational formulation, which included consideration of orbital relaxation, was first given by Koch et al. ${ }^{17}$ in their description of the first implementation of the CCSD molecular Hessian. The variational property of the CC Lagrangian and the use of the $2 n+1$ and $2 n+2$ rules gives a very compact formulation of the MBPT energy expansion that we use to identify the connected triples energy contributions in fourth and fifth orders and therefore to characterize the $\operatorname{CCSD}(\mathrm{T})$ model. $\operatorname{CCSD}(\mathrm{T})$ can be interpreted as an approximate triples model where the important connected triples are projected onto the single double space. The first iteration of CCSDT-1a can be used to obtain $\operatorname{CCSD}(\mathrm{T})$. In a similar way the first iteration of $\mathrm{CC} 3$ can be used to obtain the perturbative model $\mathrm{CC}(3)$. Energetically $\mathrm{CC}(3)$ is very close to $\operatorname{CCSD}(\mathrm{T})$. 


\section{THE APPROXIMATE COUPLED CLUSTER TRIPLES MODEL CC3}

\section{A. Coupled cluster ansatz}

The coupled cluster wave function $|\mathrm{CC}\rangle$ can be written as an exponential of a cluster operator $T$ acting on a singledeterminant wave function of noninteracting electrons,

$$
|\mathrm{CC}\rangle=\exp (T)|\mathrm{HF}\rangle \text {. }
$$

The reference wave function corresponds to the HartreeFock state $|\mathrm{HF}\rangle$. For an $N$-electron system, the cluster operator is a sum of electron excitations defined with respect to the Hartree-Fock state,

$$
T=T_{1}+T_{2}+T_{3}+\cdots+T_{N},
$$

with

$$
\begin{aligned}
& T_{1}=\sum_{a i} t_{i}^{a} a_{a}^{\dagger} a_{i}, \\
& T_{2}=\frac{1}{4} \sum_{a i b j} t_{i j}^{a b} a_{a}^{\dagger} a_{i} a_{b}^{\dagger} a_{j}, \\
& T_{3}=\frac{1}{36} \sum_{a i b j c k} t_{i j k}^{a b c} a_{a}^{\dagger} a_{i} a_{b}^{\dagger} a_{j} a_{c}^{\dagger} a_{k} .
\end{aligned}
$$

The labels $i j k \cdots$ and $a b c \cdots$ are used for occupied and unoccupied spin orbitals in the Hartree-Fock reference determinant and pqrs $\cdots$ are used as labels for orbitals with unspecified occupation. The cluster operator truncates at excitation level $N$, the number of electrons in the system. In a shorthand notation, the cluster operator is written as

$$
T=\sum_{i=1, N} T_{i}=\sum_{i=1, N} t_{\mu i} \tau_{\mu i},
$$

where the $t_{\mu_{i}}$ are the cluster amplitudes of excitation level $i$, and $\tau_{\mu_{i}}$ the associated excitation operators. The $\mathrm{CC}$ wave function satisfies the Schrödinger equation

$$
\exp (-T) H \exp (T)|\mathrm{HF}\rangle=E|\mathrm{HF}\rangle,
$$

and the coupled cluster energy is obtained by projecting Eq. (7) against $\langle\mathrm{HF}|$,

$$
E=\langle\mathrm{HF}|H \exp (T)| \mathrm{HF}\rangle .
$$

The $\mathrm{CC}$ amplitudes are determined by projecting Eq. (7) onto the excitation manifold $\left\langle\mu_{i}\right|$,

$$
\left\langle\mu_{i}|\exp (-T) H \exp (T)| \mathrm{HF}\right\rangle=0,
$$

where

$$
\left\langle\mu_{i}\right|=\langle\mathrm{HF}| \tau_{\mu_{i}}^{\dagger} .
$$

With no truncation in the cluster operator, Eqs. (8) and (9) give the full configuration interaction (FCI) solution. For truncated manifolds, the Schrödinger equation is solved in the projected space corresponding to the considered excitation level. For example, in the $\mathrm{CC}$ singles and doubles (CCSD) model, the wave function contains $T_{1}$ and $T_{2}$ and the amplitudes are determined by projecting against the singles and doubles space. The CCSDT model contains the operators $T_{1}, T_{2}$, and $T_{3}$, and the amplitudes are determined by including triples in the projection manifold. Total energies are obtained from Eq. (8).

\section{B. Coupled-cluster derivation of many-body perturbation theory}

\section{The coupled cluster Lagrangian}

The CC energy is determined from Eq. (8) subject to the constraint that the cluster amplitudes are obtained from the amplitude equations, Eq. (9). The CC energy may therefore formally be determined by an unconstrained optimization of the Lagrangian,

$$
\begin{aligned}
L(t, \bar{t})= & \langle\mathrm{HF}|H \exp (T)| \mathrm{HF}\rangle \\
& +\sum_{\mu} \bar{t}_{\mu}\langle\mu|\exp (-T) H \exp (T)| \mathrm{HF}\rangle,
\end{aligned}
$$

where both the cluster amplitudes $t_{\mu}$ and the associated Lagrange multipliers $\overline{t_{\mu}}$ are variational. There is no advantage in using the CC Lagrangian to calculate the total energy. For this purpose the simplest is to calculate the amplitudes from Eq. (9) first, and then obtain the energy from Eq. (8). However, the variational property of the parameters in Eq. (11) makes the Lagrangian convenient for evaluating a perturbation expansion of the energy. A coupled cluster formulation of many body perturbation theory has the advantage of giving expressions that are size extensive term by term. This is in contrast to Møller-Plesset perturbation theory, where the energy is size extensive order by order, but where the energy to a given order contains spurious nonsize extensive terms that cancel each other. The MBPT has been formulated in terms of diagrammatic methods giving expressions that are size extensive term by term. A coupled cluster formulation of many body perturbation theory requires no additional rules or theory.

We shall derive the perturbation expressions where the zeroth-order Hamiltonian $F$ corresponds to the Fock operator and the perturbation operator $U$ is the fluctuation potential:

$$
\begin{aligned}
& H=F+U, \\
& F=\sum_{p q}\left(h_{p q}+V_{p q}\right) a_{p}^{\dagger} a_{q}=\Sigma \epsilon_{p} a_{p}^{\dagger} a_{p}, \\
& U=\sum_{p q r s} g_{p r q s} a_{p}^{\dagger} a_{q}^{\dagger} a_{s} a_{r}-\sum_{p q} V_{p q} a_{p}^{\dagger} a_{q} .
\end{aligned}
$$

Here the $\epsilon_{p}$ are the orbital energies and $V$ the Fock potential. The CC Lagrangian now reads as

$$
\begin{aligned}
L(t, \bar{t})= & \langle\mathrm{HF}|\exp (-T) F \exp (T)| \mathrm{HF}\rangle+\left\langle\mathrm{HF}\left|U_{T}\right| \mathrm{HF}\right\rangle \\
& +\langle\bar{t}|\exp (-T) F \exp (T)| \mathrm{HF}\rangle+\left\langle\bar{t}\left|U_{T}\right| \mathrm{HF}\right\rangle,
\end{aligned}
$$

where we have introduced the notation

$$
\begin{aligned}
& U_{T}=\exp (-T) U \exp (T), \\
& \langle\bar{t}|=\sum_{\mu} \bar{t}_{\mu}\langle\mu|,
\end{aligned}
$$


assuming real parameters. We note that the commutators between $F$ and $\tau_{\mu}$ are particularly simple

$$
\left[F, \tau_{\mu}\right]=\epsilon_{\mu} \tau_{\mu} .
$$

Here $\epsilon_{\mu}$ is a linear combination of orbital energies, for example

$$
\left[F, \tau_{i j}^{a b}\right]=\left(\epsilon_{a}+\epsilon_{b}-\epsilon_{i}-\epsilon_{j}\right) \tau_{i j}^{a b} .
$$

Therefore, we obtain

$$
[F, T]=\sum_{\mu} \epsilon_{\mu} t_{\mu} \tau_{\mu},
$$

and all higher commutators vanish:

$$
[[F, T], T]=[[[F, T], T], T]=\cdots=0 .
$$

We may thus write the transformed unperturbed Hamiltonian in the form

$$
\exp (-T) F \exp (T)=F+\sum_{\mu} \epsilon_{\mu} t_{\mu} \tau_{\mu},
$$

and obtain the following (transition) expectation values:

$$
\begin{aligned}
\langle\mathrm{HF}|\exp (-T) F \exp (T)| \mathrm{HF}\rangle & =\langle\mathrm{HF}|F| \mathrm{HF}\rangle=E_{0}, \\
\langle\bar{t}|\exp (-T) F \exp (T)| \mathrm{HF}\rangle & =\sum_{\mu} \epsilon_{\mu} t_{\mu}\left\langle\bar{t}\left|\tau_{\mu}\right| \mathrm{HF}\right\rangle \\
& =\sum_{\mu \nu} \epsilon_{\mu} t_{\mu} \overline{t_{\nu}}\langle\nu \mid \mu\rangle \\
& =\sum_{\mu} \epsilon_{\mu} t_{\mu} \overline{t_{\mu}} .
\end{aligned}
$$

Inserting Eqs. (23) and (24) in the Lagrangian, Eq. (15), we obtain the following expression:

$$
L(t, \bar{t})=E_{0}+\sum_{\mu} \epsilon_{\mu} t_{\mu} \bar{t}_{\mu}+\left\langle\mathrm{HF}\left|U_{T}\right| \mathrm{HF}\right\rangle+\left\langle\bar{t}\left|U_{T}\right| \mathrm{HF}\right\rangle .
$$

We shall use this expression for the Lagrangian in our perturbation analysis of the coupled-cluster energy.

\section{The coupled-cluster variational conditions}

Since the amplitudes and multipliers are variational in the Lagrangian, they can be obtained from the variational conditions

$$
\begin{gathered}
L_{\mu}=\frac{\partial L}{\partial \bar{t}_{\mu}}=0, \\
\bar{L}_{\mu}=\frac{\partial L}{\partial t_{\mu}}=0,
\end{gathered}
$$

which hold for all perturbational strengths. Before evaluating these derivatives, we note that

$$
\frac{\partial U_{T}}{\partial t_{\mu}}=\left[U_{T}, \tau_{\mu}\right] .
$$

TABLE I. The $2 n+1$ rule for the amplitudes and the $2 n+2$ rules for the Langrange multipliers.

\begin{tabular}{ccccccc}
\hline \hline & $E^{(0)}$ & $E^{(1)}$ & $E^{(2)}$ & $E^{(3)}$ & $E^{(4)}$ & $E^{(5)}$ \\
\hline$t^{(i)}$ & 0 & 0 & 1 & 1 & 2 & 2 \\
$\bar{t}^{(i)}$ & 0 & 0 & 0 & 1 & 1 & 2 \\
\hline \hline
\end{tabular}

We now obtain the following expressions for the differentiated Lagrangian:

$$
\begin{aligned}
& L_{\mu}=\epsilon_{\mu} t_{\mu}+\left\langle\mu\left|U_{T}\right| \mathrm{HF}\right\rangle \\
& \bar{L}_{\mu}=\epsilon_{\mu} \bar{t}_{\mu}+\left\langle\mathrm{HF}\left|U_{T}\right| \mu\right\rangle+\left\langle\bar{t}\left|\left[U_{T}, \tau_{\mu}\right]\right| \mathrm{HF}\right\rangle .
\end{aligned}
$$

Except for the last term in Eq. (30), the variational conditions for the amplitudes and the associated multipliers are identical.

\section{Perturbation expansion of the coupled-cluster energy}

We wish to determine an expansion of the coupled cluster energy,

$$
E=E^{(0)}+E^{(1)}+E^{(2)}+\cdots,
$$

by expanding the Lagrangian, Eq. (25), in powers of the perturbation. In addition to the linear and explicit dependence on $U$, the Lagrangian, Eq. (25), depends implicitly and nonlinearly on the fluctuation potential through the amplitudes and the associated multipliers:

$$
\begin{aligned}
& t=t^{(0)}+t^{(1)}+t^{(2)}+\cdots, \\
& \bar{t}=\bar{t}^{(0)}+\bar{t}^{(1)}+\bar{t}^{(2)}+\cdots .
\end{aligned}
$$

The perturbation dependence of the amplitudes and their multipliers is obtained by expanding the variational conditions, Eqs. (26) and (27), in orders in $U$,

$$
\begin{aligned}
& L_{\mu}=L_{\mu}^{(0)}+L_{\mu}^{(1)}+L_{\mu}^{(2)}+\cdots, \\
& \bar{L}_{\mu}=\bar{L}_{\mu}^{(0)}+\bar{L}_{\mu}^{(1)}+\bar{L}_{\mu}^{(2)}+\cdots .
\end{aligned}
$$

An order-by-order solution of the so-called response equations,

$$
\begin{aligned}
& L_{\mu}^{(0)}=L_{\mu}^{(1)}=L_{\mu}^{(2)}=\cdots=0, \\
& \breve{L}_{\mu}^{(0)}=\breve{L}_{\mu}^{(1)}=\breve{L}_{\mu}^{(2)}=\cdots=0,
\end{aligned}
$$

yields the perturbed amplitudes and multipliers, Eqs. (32) and (33). We are interested in the energy equation (31) to fifth order. According to the $2 n+1$ rule for the amplitudes and the $2 n+2$ rule for the multipliers, we must then determine the responses of the amplitudes and the multipliers to second order. The $2 n+1$ and $2 n+2$ rules are illustrated in Table I. For a proof of the $2 n+2$ rule for the Lagrange multipliers, see Ref. 13.

\section{Coupled-cluster response equations}

To calculate the energy to fifth order, we must solve the response equations (36) and (37) to second order. The zeroth-order equations become 


$$
\begin{aligned}
& L_{\mu}^{(0)}=0 \Rightarrow \epsilon_{\mu} t_{\mu}^{(0)}=0, \\
& \bar{L}_{\mu}^{(0)}=0 \Rightarrow \epsilon_{\mu} \bar{t}_{\mu}^{(0)}=0,
\end{aligned}
$$

which means that the zeroth-order amplitudes and multipliers vanish:

$$
t_{\mu}^{(0)}=\bar{t}_{\mu}^{(0)}=0 .
$$

This fact will simplify higher-order expressions greatly. The first-order response equations are given by

$$
\begin{aligned}
& L_{\mu}^{(1)}=0 \Rightarrow \epsilon_{\mu} t_{\mu}^{(1)}+\langle\mu|U| \mathrm{HF}\rangle=0, \\
& \bar{L}_{\mu}^{(1)}=0 \Rightarrow \epsilon_{\mu} \bar{t}_{\mu}^{(1)}+\langle\mathrm{HF}|U| \mu\rangle=0 .
\end{aligned}
$$

Since these equations are the complex conjugates of each other and since real wave functions are assumed, we conclude that the first-order amplitudes and multipliers are identical:

$$
\bar{t}_{\mu}^{(1)}=t_{\mu}^{(1)} .
$$

Furthermore, since

$$
\langle\mu|U| \mathrm{HF}\rangle=\langle\mu|H| \mathrm{HF}\rangle
$$

and since the Brillouin conditions holds for the HartreeFock state, we conclude that first-order amplitudes and multipliers involve double excitations only.

According to the $2 n+1$ rule, the first-order responses determine the energy to third order. To calculate the fourth and fifth order energies, we also need the second-order responses. We therefore collect terms to second order in the variational conditions and obtain

$$
\begin{aligned}
L_{\mu}^{(2)}=0 \Rightarrow & \epsilon_{\mu} t_{\mu}^{(2)}+\left\langle\mu\left|\left[U, T^{(1)}\right]\right| \mathrm{HF}\right\rangle=0, \\
\bar{L}_{\mu}^{(2)}=0 \Rightarrow & \epsilon_{\mu} \bar{t}_{\mu}^{(2)}+\left\langle\mathrm{HF}\left|\left[U, T^{(1)}\right]\right| \mu\right\rangle \\
& +\left\langle\bar{t}^{(1)}\left|\left[U, \tau_{\mu}\right]\right| \mathrm{HF}\right\rangle=0,
\end{aligned}
$$

where we use the notation

$$
T^{(n)}=\sum_{\mu} t_{\mu}^{(n)} \tau_{\mu}
$$

We note that since $\left[U, T^{(1)}\right]$ is a rank-three operator, $\left\langle\mu\left|\left[U, T^{(1)}\right]\right| \mathrm{HF}\right\rangle$ in Eq. (45) can involve no higher than triple excitations. Therefore, the second-order amplitudes contain single, double, and triple excitations only. The second-order multipliers, in contrast, involve single, double, triple, and quadruple excitations. To see this, we note that in Eq. (46) the second term vanishes for all excitations but the last term gives nonvanishing contributions for singles, doubles, triples, and quadruples. Quintuple and higher excitations $\tau_{\mu}$ make no contribution in Eq. (46) since $\left[U, \tau_{\mu}\right]|\mathrm{HF}\rangle$ in such cases corresponds to excitations higher than doubles.

We now show that Eq. (45) and Eq. (46) are identical for the single, double, and triple excitation components, differing only in the quadruple excitation component. Expanding the commutators, we may write the second-order response equations in the form

$$
\epsilon_{\mu} t_{\mu}^{(2)}=-\left\langle\mu|U| t^{(1)}\right\rangle+\left\langle\mu\left|T^{(1)} U\right| \mathrm{HF}\right\rangle,
$$

$$
\epsilon_{\mu} \bar{t}_{\mu}^{(2)}=-\left\langle\bar{t}^{(1)}|U| \mu\right\rangle+\left\langle\bar{t}^{(1)}\left|\tau_{\mu} U\right| \mathrm{HF}\right\rangle,
$$

where

$$
\left|t^{(1)}\right\rangle=T^{(1)}|\mathrm{HF}\rangle=\sum_{\mu} t_{\mu}^{(1)}|\mu\rangle .
$$

Further simplifications are possible by invoking the resolution of identity. In Eq. (48) we thus write

$$
\begin{aligned}
\left\langle\mu\left|T^{(1)} U\right| \mathrm{HF}\right\rangle & =\left\langle\mu\left|T^{(1)}\right| \mathrm{HF}\right\rangle\langle\mathrm{HF}|U| \mathrm{HF}\rangle \\
& =\sum_{\nu} t_{\nu}^{(1)}\langle\mu \mid \nu\rangle\langle\mathrm{HF}|U| \mathrm{HF}\rangle \\
& =t_{\mu}^{(1)}\langle\mathrm{HF}|U| \mathrm{HF}\rangle .
\end{aligned}
$$

Note that only the Hartree-Fock state gives a nonvanishing contribution in the resolution of identity. The Brillouin condition eliminates all terms in the resolution of identity except the Hartree-Fock state and the doubles, and doubles do not contribute since the $\langle\mu|$ in Eq. (48) represent no higher than triple excitations. We may also simplify Eq. (49) since for all excitations $\tau_{\mu}$ we obtain

$$
\begin{aligned}
\left\langle\bar{t}^{(1)}\left|\tau_{\mu} U\right| \mathrm{HF}\right\rangle & =\left\langle\bar{t}^{(1)}\left|\tau_{\mu}\right| \mathrm{HF}\right\rangle\langle\mathrm{HF}|U| \mathrm{HF}\rangle \\
& =\bar{t}_{\mu}^{(1)}\langle\mathrm{HF}|U| \mathrm{HF}\rangle .
\end{aligned}
$$

The second-order response equations may now be written in the form

$$
\begin{aligned}
& \epsilon_{\mu} t \underset{\mu}{(2)}=-\left\langle\mu|\bar{U}| t^{(1)}\right\rangle, \\
& \epsilon_{\mu} \bar{t}_{\mu}^{(2)}=-\left\langle\bar{t}^{(1)}|\bar{U}| \mu\right\rangle,
\end{aligned}
$$

where

$$
\bar{U}=U-\langle\mathrm{HF}|U| \mathrm{HF}\rangle .
$$

These equations are the complex conjugate of each other since $\bar{t}_{\mu}^{(1)}=t_{\mu}^{(1)}$. Therefore, the second-order single, double, and triple amplitudes and multipliers are identical to each other. For quadruples, the situation is quite different since the second-order quadruple amplitudes are zero while the second-order multipliers are nonzero and are obtained from Eq. (54). We point out that it is the form of the second-order equations in Eq. (45) and (46) in terms of commutators between $U$ and the excitation operator that is needed to have each term in the energy expansion be connected. When $\bar{U}$ is introduced in Eqs. (53)-(55), disconnected energy terms will occur that are cancelled out when Eqs. (45) and (46) are used.

\section{Perturbed energies}

Having derived the equations that determine the $\mathrm{CC}$ amplitudes and multipliers to second order, we turn to the energies. The contributions from the amplitudes and multipliers to the energies (in terms of orders of perturbation) are listed in Table I. Since the excitation levels contained in the firstand second-order amplitudes and multipliers are now known, we may list the contributions from the amplitudes and multipliers to the MBPT energies; see Table II. We note that 
TABLE II. Contributions from connected amplitudes and multipliers to MBPT energies.

\begin{tabular}{lccccc}
\hline \hline Energy & $E^{(1)}$ & $E^{(2)}$ & $E^{(3)}$ & $E^{(4)}$ & $E^{(5)}$ \\
Excitations & $\mathrm{HF}$ & $D$ & $D$ & SDT & SDTQ \\
\hline \hline
\end{tabular}

singles and connected triples make their first appearance in the fourth-order energy, and that connected quadruples make a fifth-order contribution.

To determine the coupled-cluster energies to a given order, we first expand the Lagrangian equation (25) to the same order. Since the Lagrangian is variational, the resulting expansion may be simplified considerably by taking into account the variational conditions to first order Eqs. (41) and (42) and to second order Eqs. (45) and (46). The zero- and first-order energies are particularly simple, however, and straightforward expansion of the Lagrangian Eq. (25) yields

$$
\begin{aligned}
& E^{(0)}=E_{0}=\sum_{i} \epsilon_{i}, \\
& E^{(1)}=\langle\mathrm{HF}|U| \mathrm{HF}\rangle,
\end{aligned}
$$

which means that the Hartree-Fock energy is recovered to first-order in perturbation theory:

$$
E_{\mathrm{HF}}=E^{(0)}+E^{(1)} \text {. }
$$

In accordance with the $2 n+1$ and $2 n+2$ rules, there are no first-order contributions from the amplitudes and multipliers to the first-order energy.

We now consider the second-order energy. Expansion of the Lagrangian equation (25) yields the following expression:

$$
\begin{aligned}
E^{(2)}= & \sum_{\mu} \epsilon_{\mu} t_{\mu}^{(1)} \bar{t}_{\mu}^{(1)}+\left\langle\mathrm{HF}\left|\left[U, T^{(1)}\right]\right| \mathrm{HF}\right\rangle \\
& +\left\langle\bar{t}^{(1)}|U| \mathrm{HF}\right\rangle .
\end{aligned}
$$

According to the $2 n+2$ rule, the first-order multipliers make no contribution to the second-order energy. We should therefore be able to eliminate from the energy all terms that involve $\bar{t}^{(1)}$. Indeed, we note that Eq. (59) may be written in the form

$$
E^{(2)}=\sum_{\mu} L_{\mu}^{(1)} \bar{t}_{\mu}^{(1)}+\left\langle\mathrm{HF}|U| t^{(1)}\right\rangle
$$

and since the condition $L_{\mu}^{(1)}=0$ holds [see Eq. (41)], we arrive at the simple expression

$$
E^{(2)}=\left\langle\mathrm{HF}|U| t^{(1)}\right\rangle,
$$

in agreement with the $2 n+1$ and $2 n+2$ rules. The secondorder energy thus depends on the connected doubles only.

We now turn to the third-order energy. Collecting all terms to third order in the Lagrangian equation (25), we obtain

$$
\begin{aligned}
E^{(3)}= & \sum_{\mu} \epsilon_{\mu} t_{\mu}^{(1)} \bar{t}_{\mu}^{(2)}+\sum_{\mu} \epsilon_{\mu} t_{\mu}^{(2)} \bar{t}_{\mu}^{(1)} \\
& +\left\langle\mathrm{HF}\left|\left[U, T^{(2)}\right]\right| \mathrm{HF}\right\rangle \\
& +\frac{1}{2}\left\langle\mathrm{HF}\left|\left[\left[U, T^{(1)}\right], T^{(1)}\right]\right| \mathrm{HF}\right\rangle \\
& +\left\langle\bar{t}^{(1)}\left|\left[U, T^{(1)}\right]\right| \mathrm{HF}\right\rangle+\left\langle\bar{t}^{(2)}|U| \mathrm{HF}\right\rangle
\end{aligned}
$$

This expression may be simplified considerably. First we note that the fourth term vanishes since the double commutator is a rank four operator containing double or higher excitations. Next, according to the $2 n+1$ and $2 n+2$ rules, $E^{(3)}$ does not involve second-order amplitudes and mutlipliers. Indeed, we find that Eq. (62) may be written in the form

$$
E^{(3)}=\sum_{\mu} L_{\mu}^{(1)} \bar{t}_{\mu}^{(2)}+\sum_{\mu} \bar{L}_{\mu}^{(1)} t_{\mu}^{(2)}+\left\langle\bar{t}^{(1)}\left|\left[U, T^{(1)}\right]\right| \mathrm{HF}\right\rangle,
$$

which reduces to the expression

$$
E^{(3)}=\left\langle\bar{t}^{(1)}\left|\left[U, T^{(1)}\right]\right| \mathrm{HF}\right\rangle,
$$

by invoking the first-order variational conditions, Eqs. (41) and (42), in agreement with the $2 n+1$ rule. Equation (64) may be rewritten by expanding the commutator and introducing the resolution of identity, yielding

$$
E^{(3)}=\left\langle\bar{t}^{(1)}|\bar{U}| t^{(1)}\right\rangle .
$$

Equation (65) is the form that is obtained in conventional Rayleigh Schrödinger perturbation theory. It contains disconnected energy contributions in contrast to Eq. (64) where the commutator ensures that all energy contributions are connected.

As indicated in Table II, the fourth-order energy should involve contributions from singles, doubles, and triples. To derive the detailed form of this energy, we expand the Lagrangian to fourth order:

$$
\begin{aligned}
E^{(4)}= & \sum_{\mu} \epsilon_{\mu} t_{\mu}^{(1)} \bar{t}_{\mu}^{(3)}+\sum_{\mu} \epsilon_{\mu} t_{\mu}^{(2)} \bar{t}_{\mu}^{(2)}+\sum_{\mu} \epsilon_{\mu} t_{\mu}^{(3)} \bar{t}_{\mu}^{(1)} \\
& +\left\langle\mathrm{HF}\left|\left[U, T^{(3)}\right]\right| \mathrm{HF}\right\rangle+\left\langle\mathrm{HF}\left|\left[\left[U, T^{(2)}\right], T^{(1)}\right]\right| \mathrm{HF}\right\rangle \\
& +\frac{1}{6}\left\langle\mathrm{HF}\left|\left[\left[\left[U, T^{(1)}\right], T^{(1)}\right], T^{(1)}\right]\right| \mathrm{HF}\right\rangle \\
& +\left\langle\bar{t}^{(1)}\left|\left[U, T^{(2)}\right]\right| \mathrm{HF}\right\rangle \\
& +\frac{1}{2}\left\langle\bar{t}^{(1)}\left|\left[\left[U, T^{(1)}\right], T^{(1)}\right]\right| \mathrm{HF}\right\rangle \\
& +\left\langle\bar{t}^{(2)}\left|\left[U, T^{(1)}\right]\right| \mathrm{HF}\right\rangle+\left\langle\bar{t}^{(3)}|U| \mathrm{HF}\right\rangle .
\end{aligned}
$$

The fifth and sixth terms in this expression vanish since the commutators involve too high excitation levels. The energy may therefore be written in the simpler form:

$$
\begin{aligned}
E^{(4)}= & \sum_{\mu} L_{\mu}^{(1)} \bar{t}_{\mu}^{(3)}+\sum_{\mu} L_{\mu}^{(2)} \bar{t}_{\mu}^{(2)}+\sum_{\mu} t_{\mu}^{(3)} \bar{L}_{\mu}^{(1)} \\
& +\left\langle\bar{t}^{(1)}\left|\left[U, T^{(2)}\right]\right| \mathrm{HF}\right\rangle \\
& +\frac{1}{2}\left\langle\bar{t}^{(1)}\left|\left[\left[U, T^{(1)}\right], T^{(1)}\right]\right| \mathrm{HF}\right\rangle,
\end{aligned}
$$

which yields (invoking the variational conditions) 


$$
E^{(4)}=\left\langle\bar{t}^{(1)}\left|\left[U, T^{(2)}\right]\right| \mathrm{F}\right\rangle+\frac{1}{2}\left\langle\bar{t}^{(1)}\left|\left[\left[U, T^{(1)}\right], T^{(1)}\right]\right| \mathrm{HF}\right\rangle,
$$

in accordance with the $2 n+1$ and $2 n+2$ rules. Since $t^{(2)}$ does not have quadruple contributions, the fourth-order energy does not have contributions from connected quadruples. The commutators in Eq. (68) ensure that all energy contributions are connected. Eq. (68) may be rewritten to the form that is obtained in conventional Rayleigh Schrödinger perturbation theory and that contains disconnected energy contributions. To do this, we expand the ommutators, yielding

$$
E^{(4)}=\left\langle\bar{t}(1)|\bar{U}| t^{(2)}-\frac{1}{2} t^{(1)} t^{(1)}\right\rangle-\left\langle\bar{t}^{(1)} \mid t^{(1)}\right\rangle E^{(2)},
$$

where we use the notation

$$
\left|t^{(2)}-\frac{1}{2} t^{(1)} t^{(1)}\right\rangle=\left(T^{(2)}-\frac{1}{2} T^{(1)} T^{(1)}\right)|\mathrm{HF}\rangle .
$$

The fourth-order energy in Eq. (69) arises from interactions of connected doubles with singles, connected doubles, connected triples, disconnected quadruples, and also the Hartree-Fock state. There are no contributions from connected quadruples. Also, there are no interactions that do not involve connected doubles.

Proceeding finally to fifth order, we obtain the expression

$$
\begin{aligned}
E^{(5)}= & \sum_{\mu} L_{\mu}^{(1)} \bar{t}_{\mu}^{(4)}+\sum_{\mu} L_{\mu}^{(2)} \bar{t}_{\mu}^{(3)}+\sum_{\mu} t_{\mu}^{(3)} \bar{L}_{\mu}^{(2)} \\
& +\sum_{\mu} t_{\mu}^{(4)} \bar{L}_{\mu}^{(1)}+\frac{1}{2}\left\langle\mathrm{HF}\left|\left[\left[U, T^{(2)}\right], T^{(2)}\right]\right| \mathrm{HF}\right\rangle \\
& +\left\langle\bar{t}^{(1)}\left|\left[\left[U, T^{(2)}\right], T^{(1)}\right]\right| \mathrm{HF}\right\rangle \\
& +\left\langle\bar{t}^{(2)}\left|\left[U, T^{(2)}\right]\right| \mathrm{HF}\right\rangle \\
& +\frac{1}{2}\left\langle\bar{t}^{(2)}\left|\left[\left[U, T^{(1)}\right], T^{(1)}\right]\right| \mathrm{HF}\right\rangle,
\end{aligned}
$$

and by invoking the first and second-order variational conditions we arrive at the expression

$$
\begin{aligned}
E^{(5)}= & \frac{1}{2}\left\langle\mathrm{HF}\left|\left[\left[U, T^{(2)}\right], T^{(2)}\right]\right| \mathrm{HF}\right\rangle \\
& +\left\langle\bar{t}^{(1)}\left|\left[\left[U, T^{(2)}\right], T^{(1)}\right]\right| \mathrm{HF}\right\rangle \\
& +\left\langle\bar{t}^{(2)}\left|\left[U, T^{(2)}\right]\right| \mathrm{HF}\right\rangle \\
& +\frac{1}{2}\left\langle\bar{t}^{(2)}\left|\left[\left[U, T^{(1)}\right], T^{(1)}\right]\right| \mathrm{HF}\right\rangle,
\end{aligned}
$$

for the fifth-order energy, in agreement with the $2 n+1$ rule. Clearly, we have contributions from connected quadruples in the terms involving $t^{(2)}$. Using the coupled cluster parametrization of the wave function, we obtain directly that all energy contributions are connected.

\section{The $\operatorname{CCSD}(T)$ model}

Since the CCSD model contains singles and doubles only, it is correct to third order in perturbation theory. To higher orders connected triples make their appearance and any improvement on the CCSD model must take into account the effect of these excitations. Let us identify all en- ergy contributions from connected triples to fourth and fifth orders in the perturbation. The connected triples give one fourth-order contribution

$$
E_{T}^{(4)}=\left\langle\bar{t}_{2}^{(1)}\left|\left[U, T_{3}^{(2)}\right]\right| \mathrm{HF}\right\rangle,
$$

where, for example, $T_{3}^{(2)}$ represents the part of $T^{(2)}$ containing triples. In fifth order, several contributions arise. From a consideration of excitation levels, the first two terms in Eq. (72) are seen not to contain contributions from connected triples. The remaining two terms give rise to the following fifthorder connected triples contributions:

$$
\begin{aligned}
E_{T}^{(5)}= & \left\langle\bar{t}_{1}^{(2)}\left|\left[U, T_{3}^{(2)}\right]\right| \mathrm{HF}\right\rangle+\left\langle\bar{t}_{2}^{(2)}\left|\left[U, T_{3}^{(2)}\right]\right| \mathrm{HF}\right\rangle \\
& +\left\langle\bar{t}_{3}^{(2)}\left|\left[U, T_{3}^{(2)}\right]\right| \mathrm{HF}\right\rangle+\left\langle\bar{t}_{4}^{(2)}\left|\left[U, T_{3}^{(2)}\right]\right| \mathrm{HF}\right\rangle \\
& +\left\langle\bar{t}_{3}^{(2)}\left|\left[U, T_{2}^{(2)}\right]\right| \mathrm{HF}\right\rangle \\
& +\frac{1}{2}\left\langle\bar{t}_{3}^{(2)}\left|\left[\left[U, T_{2}^{(1)}\right], T_{2}^{(1)}\right]\right| \mathrm{HF}\right\rangle .
\end{aligned}
$$

Connected triples thus give a fourth-order contribution when projected against the doubles space and fifth-order contributions when projected against the singles, doubles, triples, and quadruples space.

The $E_{T}^{(4)}$ term in Eq. (73) and the first two terms in $E_{T}^{(5)}$ of Eq. (74) are the only fourth- and fifth-order contributions that result from the projection of connected triples onto the singles and doubles space. For singles and doubles we may write

$$
\left\langle t^{\mathrm{CCSD}}\right|=\left\langle\bar{t}_{1}^{(2)}\right|+\left\langle\bar{t}_{2}^{(1)}\right|+\left\langle\bar{t}_{2}^{(2)}\right|+O\left(t_{1}^{(3)}, t_{2}^{(3)}\right) .
$$

We may therefore account for the effects of the fourth- and fifth-order triples projected against singles and doubles by calculating the following correction to the CCSD energy: ${ }^{8}$

$$
E_{\text {corr }}^{\mathrm{CCSS}(\mathrm{T})}=\left\langle t^{\mathrm{CCSD}}\left|\left[U, T_{3}^{(2)}\right]\right| \mathrm{HF}\right\rangle .
$$

The terms differing from the true MBPT expansion because of the use of $\left\langle t^{\mathrm{CCSD}}\right|$ rather than $\left\langle\bar{t}_{1}^{(2)}+\bar{t}_{2}^{(1)}+\bar{t}_{2}^{(2)}\right|$ are at least of sixth order and are thus of little importance relative to the remaining fifth-order terms in Eq. (74).

\section{The CC3 model}

Approximate coupled cluster triples models may alternatively be obtained by simplifying the cluster amplitude equations of the CCSDT model and iterating these until convergence. Here we present one such approximate scheme, referred to as the $\mathrm{CC} 3$ model, where approximations are guided by the following requirements: (1) the triples amplitudes should be correct to the first nonvanishing order in perturbation theory; and (2) the singles amplitudes should be treated as zeroth-order parameters because of their unique role as orbital relaxation parameters. The CCSDT amplitude equations may be written as

$$
\begin{aligned}
& \left\langle\mu_{1}\left|\exp \left(-T_{1}-T_{2}-T_{3}\right) H \exp \left(T_{1}+T_{2}+T_{3}\right)\right| \mathrm{HF}\right\rangle=0, \\
& \left\langle\mu_{2}\left|\exp \left(-T_{1}-T_{2}-T_{3}\right) H \exp \left(T_{1}+T_{2}+T_{3}\right)\right| \mathrm{HF}\right\rangle=0,
\end{aligned}
$$




$$
\left\langle\mu_{3}\left|\exp \left(-T_{1}-T_{2}-T_{3}\right) H \exp \left(T_{1}+T_{2}+T_{3}\right)\right| \mathrm{HF}\right\rangle=0 .
$$

Introducing the modified two-electron Hamiltonian,

$$
\hat{H}=\exp \left(-T_{1}\right) H \exp \left(T_{1}\right),
$$

we may write the singles and doubles equations in the form

$$
\begin{aligned}
& \left\langle\mu_{1}\left|\exp \left(-T_{2}\right) \hat{H} \exp \left(T_{2}\right)\right| \mathrm{HF}\right\rangle+\left\langle\mu_{1}\left|\left[H, T_{3}\right]\right| \mathrm{HF}\right\rangle=0, \\
& \left\langle\mu_{2}\left|\exp \left(-T_{2}\right) \hat{H} \exp \left(T_{2}\right)\right| \mathrm{HF}\right\rangle+\left\langle\mu_{2}\left|\left[\hat{H}, T_{3}\right]\right| \mathrm{HF}\right\rangle=0 .
\end{aligned}
$$

Expanding the triples equations (79), we obtain

$$
\begin{aligned}
& \left\langle\mu_{3}\left|\left[F, T_{3}\right]\right| \mathrm{HF}\right\rangle+\left\langle\mu_{3}\left|\left[\hat{U}, T_{2}\right]\right| \mathrm{HF}\right\rangle \\
& \quad+\frac{1}{2}\left\langle\mu_{3}\left|\left[\left[\hat{U}, T_{2}\right], T_{2}\right]\right| \mathrm{HF}\right\rangle+\left\langle\mu_{3}\left|\left[\hat{U}, T_{3}\right]\right| \mathrm{HF}\right\rangle \\
& \quad+\left\langle\mu_{3}\left|\left[\left[U, T_{3}\right], T_{2}\right]\right| \mathrm{HF}\right\rangle=0,
\end{aligned}
$$

with $H_{0}$ and $U$ defined as in Eqs. (12)-(14) and

$$
\hat{U}=\exp \left(-T_{1}\right) U \exp \left(T_{1}\right) \text {. }
$$

The computational costs of the five terms in Eq. (83) scale as $N^{6}, N^{7}, N^{7}, N^{8}$, and $N^{8}$, respectively. When the transformed operators $\hat{H}$ and $\hat{U}$ are used in Eqs. (81)-(83), the singles amplitudes are treated as zeroth-order parameters. Also, use of the first two terms in Eq. (83) ensures that the connected triples are correct to second order in perturbation theory. Thus, in the CC 3 model we determine the wave function from the singles and doubles equations Eqs. (81) and (82) together with the triples equation

$$
\left\langle\mu_{3}\left|\left[F, T_{3}\right]\right| \mathrm{HF}\right\rangle+\left\langle\mu_{3}\left|\left[\hat{U}, T_{2}\right]\right| \mathrm{HF}\right\rangle=0 .
$$

This equation ensures that the triples are correct to second order and also treats orbital relaxation in an approximate fashion. The simple form of Eq. (85) allows an explicit solution for the triples in terms of the singles and doubles amplitudes:

$$
t_{\mu_{3}}=-\epsilon_{\mu_{3}}^{-1}\left\langle\mu_{3}\left|\left[\hat{U}, T_{2}\right]\right| \mathrm{HF}\right\rangle .
$$

Introducing the notation

$$
Q_{3}=-\sum_{\mu_{3}} \epsilon_{\mu_{3}}^{-1}\left\langle\mu_{3} \mid\left[\hat{U}, T_{2}\right] \mathrm{HF}\right\rangle \tau_{\mu_{3}},
$$

for the approximate triples amplitudes, we may write the CC3 cluster amplitude equations as

$$
\begin{aligned}
& \left\langle\mu_{1}\left|\exp \left(-T_{2}\right) \hat{H} \exp \left(T_{2}\right)\right| \mathrm{HF}\right\rangle+\left\langle\mu_{1}\left|\left[H, Q_{3}\right]\right| \mathrm{HF}\right\rangle=0, \\
& \left\langle\mu_{2}\left|\exp \left(-T_{2}\right) \hat{H} \exp \left(T_{2}\right)\right| \mathrm{HF}\right\rangle+\left\langle\mu_{2}\left|\left[\hat{H}, Q_{3}\right]\right| \mathrm{HF}\right\rangle=0 .
\end{aligned}
$$

The first term in Eqs. (88) and (89) represents the contributions to the singles and doubles equations that appear in CCSD. The second term represents the nonvanishing connected triples contributions. The singles and doubles equations in Eqs. (88) and (89) are similar to the CCSDT singles and doubles equations, where $T_{3}$ is replaced by the perturbation correct form $Q_{3}$.

The CC3 model is similar in spirit to the CCSDT-n models of Urban et al. ${ }^{6}$ Thus CCSDT-1b is obtained from CC3 if $\hat{U}$ in Eq. (87) is replaced by $U$. The CCSDT-1a model is obtained if, in addition, $\hat{H}$ in the last term of the double equations, Eq. (89), is replaced by $H$. In the more elaborate CCSDT-n models $(n=2,3,4)$, various contributions from the last three terms in Eq. (83) are retained in the triples equations. We note that such approximations may become unbalanced if the terms to be included are not carefully selected according to perturbation theory:

$$
\epsilon_{\mu} t_{\mu}^{(3)}=-\left\langle\mu\left|\left[U, T^{(2)}\right]\right| \mathrm{HF}\right\rangle-\frac{1}{2}\left\langle\mu\left|\left[\left[U, T^{(1)}\right], T^{(1)}\right]\right| \mathrm{HF}\right\rangle .
$$

If both terms are included, the model scales as $N^{8}$ and it is then probably better to resort to a full CCSDT treatment, which also scales as $N^{8}$. Indeed, the results obtained by Urban et al. ${ }^{6}$ confirm that the CCSDT-2 and CCSDT- 3 may be unbalanced since they perform no better than CCSDT- $1 b$. The inclusion of the singles does not give similar problems because of the unique role of singles as approximate orbital relaxation parameters.

The $\operatorname{CCSD}(T)$ energy can be generated by carrying out a CCSD calculation and multiplying the correction term of the first CCSDT-1a iteration by the CCSD singles and doubles

TABLE III. Total energies (a.u.) in coupled cluster calculations and FCI calculations on $\mathrm{H}_{2} \mathrm{O}$ at various $\mathrm{OH}$ bond length using the cc-pVDZ basis. $W_{\mathrm{HF}}$ is the weight of the Hartree-Fock configuration in the FCI wave function.

\begin{tabular}{lccccc}
\hline \hline & $E_{e}$ & $1.5 R_{e}$ & $2 R_{e}$ & $2.5 R_{e}$ & $3 R_{e}$ \\
\hline$R_{\mathrm{HF}}$ & -76.024039 & -75.802397 & -75.587711 & -75.441244 & -75.344392 \\
$W_{\mathrm{HF}}$ & 0.941 & 0.849 & 0.589 & 0.337 & 0.230 \\
$E_{\mathrm{CCSD}}$ & -76.238116 & -76.062305 & -75.929633 & -75.897684 & -75.901097 \\
$E_{\mathrm{CCSD}(\mathrm{T})}$ & -76.241202 & -76.070717 & -75.955485 & -75.960555 & -76.002458 \\
$E_{\mathrm{CC}(3)}$ & -76.241202 & -76.070697 & -75.954928 & -75.961190 & -76.003528 \\
$E_{\mathrm{CCSDT}-1 \mathrm{a}}$ & -76.241273 & -76.070747 & -75.953780 & -75.943580 & -75.955745 \\
$E_{\mathrm{CCSDT}-1 \mathrm{~b}}$ & -76.241273 & -76.070759 & -75.953401 & -75.945461 & -75.958153 \\
$E_{\mathrm{CC} 3}$ & -76.241274 & -76.070726 & -75.952809 & -75.943671 & $\cdots$ \\
$E_{\mathrm{CCSDT}}$ & -76.241367 & -76.070925 & -75.953070 & -75.942743 & -75.952072 \\
$E_{\mathrm{FCI}}$ & -76.241860 & -76.072348 & -75.951665 & -75.917991 & $-75.911946(1)$ \\
\hline \hline
\end{tabular}


TABLE IV. Total energies (a.u.) for $\mathrm{C}_{2}$ at different internuclear distances using cc-pVDZ basis.

\begin{tabular}{ccccccc}
\hline \hline$R$ (a.u.) & CCSD & CCSD $(\mathrm{T})$ & CC $(3)$ & CCSDT-1a & CC3 & CCSDT \\
\hline 2.0 & -75.608608 & -75.635114 & -75.635473 & -75.634497 & -75.634003 & -75.633445 \\
3.0 & -75.626299 & -75.658899 & -75.662029 & -75.661521 & -75.662270 & -75.657850 \\
4.0 & -75.501214 & -75.549216 & -75.552255 & -75.543871 & -75.544332 & -75.575108 \\
5.0 & -75.482059 & -75.573037 & -75.574948 & -75.537687 & -75.537277 & -75.546522 \\
5.5 & -75.482395 & -75.592087 & -75.593459 & -75.540558 & -75.540179 & -75.548803 \\
\hline \hline
\end{tabular}

amplitudes. In a similar spirit, we introduce the $\mathrm{CC}(3)$ model, where the total energy is obtained from the CCSD energy by adding to it the term generated by multiplying the correction term from the first $\mathrm{CC} 3$ iteration by the CCSD amplitudes. Energetically, $\mathrm{CC}(3)$ does not differ much from $\operatorname{CCSD}(\mathrm{T})$

\section{E. Closed shell CC3}

In the CC3 model, Eqs. (88) and (89) are solved using the triples amplitudes generated according to Eq. (87). The total energy is obtained from Eq. (8) using the converged singles and doubles amplitudes. We now consider in greater detail a closed-shell system. Since the nonlinear amplitude equations are solved iteratively, the major computational task is to construct the vector functions in Eqs. (88) and (89) for a set of trial amplitudes. The right-hand sides in Eqs. (88) and (89) appear in standard CCSD theory for closed-shell systems and are given in Ref. 18 using the notation of this paper. The singles and doubles projection manifold is parametrized in terms of a biorthonormal basis:

$$
\begin{aligned}
& \left\langle\begin{array}{l}
a \\
i
\end{array}\right|=\langle\mathrm{HF}| E_{i a}, \\
& \left\langle\begin{array}{ll}
a & b \\
i & j
\end{array}\right|=\frac{1}{3}\langle\mathrm{HF}|\left(2 E_{j b} E_{i a}+E_{i b} E_{j a}\right),
\end{aligned}
$$

The one- and two-electron cluster operators are

$$
\begin{aligned}
& T_{1}=\sum_{a i} t_{i}^{a} E_{a i} \\
& T_{2}=\frac{1}{2} \sum_{a b i j} t_{i j}^{a b} E_{a i} E_{b j},
\end{aligned}
$$

where $t_{i j}^{a b}$ is symmetric with respect to permutations $(a i) \leftrightarrow(b j)$. The three-electron cluster operator is parametrized as

TABLE V. Error relative to CCSDT in $\mathrm{mH}$ for $\mathrm{C}_{2}$.

\begin{tabular}{lrrrrr}
\hline \hline$R$ (a.u.) & \multicolumn{1}{c}{ CCSD } & CCSD(T) & \multicolumn{1}{c}{ CC(3) } & CCSDT-1a & \multicolumn{1}{c}{ CC3 } \\
\hline 2.0 & -24.837 & 1.669 & 2.028 & 1.052 & 0.558 \\
3.0 & -31.551 & 1.049 & 4.179 & 3.671 & 4.420 \\
4.0 & -73.894 & -25.892 & -22.853 & -31.237 & -30.776 \\
5.0 & -64.462 & -26.515 & -28.426 & -8.835 & -9.245 \\
5.5 & -66.408 & -43.284 & -44.656 & -8.245 & -8.624 \\
\hline \hline
\end{tabular}

$$
T_{3}=\frac{1}{6} \sum_{a i b j c k} t_{i j k}^{a b c} E_{a i} E_{b j} E_{c k},
$$

where the amplitudes are symmetric with respect to the permutations $(a i) \leftrightarrow(b j) \leftrightarrow(c k)$. Assuming that $\left\langle\mu_{3}\right|$ refers to a biorthonormal basis, the triples amplitudes can be determined from Eq. (84) as

$$
t_{i j k}^{a b c}=-\frac{\Gamma_{i j k}^{a b c}}{\epsilon_{i j k}^{a b c}},
$$

where

$$
\begin{aligned}
& \epsilon_{i j k}^{a b c}=\epsilon_{a}+\epsilon_{b}+\epsilon_{c}-\epsilon_{i}-\epsilon_{j}-\epsilon_{k}, \\
& \Gamma_{i j k}^{a b c}=P_{i j k}^{a b c}\left\{\sum_{d} t_{i j}^{a d}(c k \hat{\mid} b d)-\sum_{l} t_{i l}^{a b}(c k \hat{\mid} l j)\right\} .
\end{aligned}
$$

In Eq. (99) $P_{i j k}^{a b c}$ is an operator that permutes the indices

$$
\begin{aligned}
P_{i j k}^{a b c}= & \left(\begin{array}{c}
a b c \\
i j k
\end{array}\right)+\left(\begin{array}{c}
a c b \\
i k j
\end{array}\right)+\left(\begin{array}{c}
b a c \\
j i k
\end{array}\right)+\left(\begin{array}{c}
c a b \\
k i j
\end{array}\right)+\left(\begin{array}{c}
b c a \\
j k i
\end{array}\right) \\
& +\left(\begin{array}{c}
c b a \\
k j i
\end{array}\right) .
\end{aligned}
$$

Using the triples amplitudes, Eq. (96), the contributions that are added to the standard CCSD amplitude equations [see Eqs. (88) and (89)] become

$$
\begin{aligned}
& \left\langle\begin{array}{l}
a \\
i
\end{array}\left|\left[H, T_{3}\right]\right| \mathrm{HF}\right\rangle=2 \sum_{b j c k}\left(t_{i j k}^{a b c}-t_{k j i}^{a b c}\right) L_{j b k c} \\
& \left\langle\left\langle_{i j}^{a b}\left|\left[\hat{H}, T_{3}\right]\right| \mathrm{HF}\right\rangle\right. \\
& =2 P_{i j}^{a b}\left\{\sum_{c k}\left(t_{i j k}^{a b c}-t_{k j i}^{a b c}\right) \hat{F}_{k c}+\sum_{c k d}\left(2 t_{j i k}^{b c d}-t_{k i j}^{b c d}-t_{j k i}^{b c d}\right)\right. \\
& \left.\quad \times(a c \hat{\mid} k d)-\sum_{c k l}\left(2 t_{j k l}^{b a c}-t_{l k j}^{b a c}-t_{j l k}^{b a c}\right)(k i \hat{\mid} \mid l c)\right\},
\end{aligned}
$$

where

$$
\begin{aligned}
& P_{i j}^{a b}=\left(\begin{array}{c}
a b \\
i j
\end{array}\right)+\left(\begin{array}{c}
b a \\
j i
\end{array}\right), \\
& \hat{F}_{p q}=\hat{h}_{p q}+\sum_{k} \hat{L}_{k k p q}, \\
& \hat{L}_{p q r s}=2(p q \hat{\mid} r s)-(p s \hat{\mid} r q),
\end{aligned}
$$


TABLE VI. Total energies (a.u.) for CO at different internuclear distances using cc-pVDZ basis.

\begin{tabular}{ccccccc}
\hline \hline$R$ (a.u.) & CCSD & CCSD(T) & CC(3) & CCSDT-1a & CC3 & CCSDT \\
\hline 1.6 & -112.641639 & -112.647129 & -112.647249 & -112.647327 & -112.647390 & -112.647390 \\
1.8 & -112.924689 & -112.931696 & -112.931935 & -112.932086 & -112.932015 & -112.932015 \\
2.0 & -113.030265 & -113.039292 & -113.039782 & -113.040113 & -113.040685 & -113.039685 \\
2.5 & -112.995571 & -113.012957 & -113.015485 & -113.018147 & -113.018608 & -113.013608 \\
3.0 & -112.861713 & -112.894160 & -112.904689 & -112.923328 & -112.920757 & -112.894757 \\
\hline \hline
\end{tabular}

and $(p q \hat{\mid} r s)$ denotes integrals for the modified Hamiltonian. We refer to Ref. 18 for details about the integrals of the modified Hamiltonian.

\section{SAMPLE CALCULATIONS}

The performance of the $\mathrm{CC} 3$ and $\mathrm{CC}(3)$ models has been tested by carrying out calculations on $\mathrm{H}_{2} \mathrm{O}, \mathrm{C}_{2}, \mathrm{CO}$, and $\mathrm{C}_{2} \mathrm{H}_{4}$. For all molecules except ethylene we stretch the bonds to investigate how well the models describe the increasing size of the static correlation contribution. For comparison we give results of the approximate triples models $\operatorname{CCSD}(\mathrm{T})$, CCSDT-1a, CCSDT-1b and full CCSDT. For water we also quote the full configuration interaction (FCI) results. ${ }^{19}$ The CCSDT calculations were carried out using the ACESII program. ${ }^{20} \mathrm{CCSD}(\mathrm{T}), \mathrm{CCSDT}-1 \mathrm{a}$, and CCSDT-1b results have been checked against ACESII results.

The water calculations are carried out in $C_{2 v}$ symmetry, using geometries from previous FCI calculations. The $\mathrm{HOH}$ bond angle is fixed as $110.6^{\circ}$ and the $\mathrm{OH}$ distances are chosen as $R_{e}, 1.5 R_{e}, 2.0 R_{e}, 2.5 R_{e}$, and $3.0 R_{e}$, with $R_{e}$ $=1.84345$ a.u. The Cartesian coordinates of these geometries are given in Ref. 19. We have used the spherical cc-pVDZ basis of Dunning ${ }^{21}$ and all ten electrons are correlated. FCI, CCSD, CCSDT, and CCSD $(\mathrm{T})$ results have previously been reported for the same geometries and basis set. The weight of the Hartree-Fock configuration in the FCI wave function $\left(W_{\mathrm{HF}}\right)$ and the FCI natural orbital occupation numbers were also reported in Ref. 19. In Table III we list the results of the approximate triples calculations CC3, CC(3), CCSDT-1a, and CCSDT-1b together with the above-mentioned results.

The approximate triples results can be divided in two groups, the iterative results CC 3 , CCSDT-1a, and CCSDT$1 \mathrm{~b}$, and the perturbative results $\mathrm{CC}(3)$ and $\operatorname{CCSD}(\mathrm{T})$. Close to the equilibrium geometry, the Hartree-Fock configuration dominates and very small differences are observed between the iterative and perturbative methods. Both the iterative and the perturbation-based models approximate very well the full

TABLE VII. Error relative to CCSDT in $\mathrm{mH}$ for CO.

\begin{tabular}{cccccr}
\hline \hline$R$ (a.u.) & CCSD & CCSD(T) & CC(3) & CCSDT-1a & CC3 \\
\hline 1.6 & -5.751 & -0.261 & -0.141 & -0.063 & -0.052 \\
1.8 & -7.326 & -0.319 & -0.080 & -0.071 & 0.084 \\
2.0 & -9.420 & -0.393 & 0.097 & 0.428 & 0.440 \\
2.5 & -18.037 & -0.651 & 1.877 & 4.539 & 4.410 \\
3.0 & -33.044 & -0.597 & 9.932 & 28.571 & 26.160 \\
\hline \hline
\end{tabular}

CCSDT results. For example, at equilibrium the largest deviation between the approximate triples and full triples result is $0.16 \mathrm{mH}$ and occurs for $\mathrm{CC}(3)$ and $\operatorname{CCSD}(\mathrm{T})$. At larger internuclear distances the perturbation models show some degradence, while the iterative approaches approximate the full CCSDT result quite well. For example, at $2 R_{e}$, where the weight of the Hartree-Fock configuration is 0.589 , the CC3 energy is $0.26 \mathrm{mH}$ above the full CCSDT energy, while the $\operatorname{CCSD}(\mathrm{T})$ energy is $2.41 \mathrm{mH}$ below CCSDT. At internuclear distances up to $2 R_{e}$ CCSDT is quite close to FCI, whereas for larger $\mathrm{OH}$ distances significant degradence occurs. Significantly, even for distances where full CCSDT behaves poorly compared to FCI do the iterative models approximate well CCSDT for example at $2.5 R_{e}$ CC3 is 0.93 $\mathrm{mH}$ below CCSDT, while CCSDT-1a and CCSDT-1b are 0.84 and $2.72 \mathrm{mH}$ below, respectively. In water CC3 thus approximates very well CCSDT, even when the static correlation is so large that the performance of CCSDT deteriorates compared to FCI.

In Table IV total energies are given for $\mathrm{C}_{2}$ in CCSD, CCSD(T), CC(3), CCSDT-1a, CC3, and CCSDT. The internuclear distance varies between 2.0 and 5.5 a.u. The ccpVDZ basis set is used. In Table V, we list the errors in CCSD and in the approximate triples models relative to CCSDT. Close to equilibrium all triples methods reduce the CCSD error by about $90 \%$, with the error now being of opposite sign. At 3.0 a.u., CC3 is the triples method with the largest error relative to CCSDT $(4 \mathrm{mH})$. In the region $4.0-$ 5.5 a.u. the errors increase significantly-the errors of the triples methods are about half the error of CCSD relative to CCSDT. For all geometries $\mathrm{CC}(3)$ is close to $\operatorname{CCSD}(\mathrm{T})$ and CCSDT-1a is close to CC3. The perturbative approaches differ significantly from the iterative approaches at some distances. Since errors of the same size occur in the interval 4.0-5.5 a.u. for both iterative and not-iterative models, no systematic trends can be identified.

For $\mathrm{CO}$ total energies have been calculated in the interval 1.6-3.0 a.u. using the cc-pVDZ basis set. The energies of the different approaches are given in Table VI, and in Table VII the errors relative to CCSDT are listed. $\operatorname{CCSD}(\mathrm{T})$ is within $0.7 \mathrm{mH}$ of CCSDT at all geometries, while the other methods show a significant degradence with increasing internuclear distance. Note that the behavior of $\mathrm{CC}(3)$ is quite different from $\operatorname{CCSD}(\mathrm{T})$. The iterative models are within 0.1 $\mathrm{mH}$ of CCSDT for $R=1.6-1.8$ a.u.

The results for ethylene at equilibrium geometry using the cc-pVDZ basis set are given in Table VIII. All approxi- 
TABLE VIII. Total energies (a.u.) for $\mathrm{C}_{2} \mathrm{H}_{4}$ using various coupled cluster models using cc-pVDZ basis.

\begin{tabular}{ll}
\hline \hline CCSD & -75.349900 \\
CCSD $(\mathrm{T})$ & -78.359864 \\
CC $(3)$ & -78.359871 \\
CCSDT-1a & -78.360069 \\
CC3 & -78.360073 \\
CCSDT & -78.360488 \\
\hline \hline
\end{tabular}

mate triples methods account for more than $90 \%$ of the effect of triples. The $\operatorname{CCSD}(\mathrm{T})$ and $\mathrm{CC}(3)$ results are very close $(0.01 \mathrm{mH})$ and the same holds for CC3 relative to CCSDT1a. The extra singles contributions in $\mathrm{CC}(3)$ and $\mathrm{CC} 3$ thus seem to be insignificant.

\section{SUMMARY}

We have developed an iterative approximate triples model, CC3, where the triples amplitudes are correct through second order, and where no approximations are made in the treatment of the singles amplitudes because of their unique role as orbital relaxation parameters.

To better understand the structure of the approximate triples models, we have given an alternative deviation of many-body perturbation theory, using the coupled cluster parametrization of the wave function and the method of undetermined Lagrange multipliers to set up a variational coupled cluster energy. In this formulation, the $n$ th-order cluster parameters and Lagrange multipliers determine the energy to order $2 n+1$. In fact, only the $n$ th-order multipliers are needed to obtain the energy to order $2 n+2$. The compact expressions obtained from the variational formulation reveal in a simple manner the connection between the iterative CC3. CCSDT-1a, and CCSDT-1b models and the perturbative $\operatorname{CCSD}(\mathrm{T})$ model. It shows that the first iteration of CCSDT-1a can in a simple manner be used to obtain the CCSD(T) energy. From the first iteration of $\mathrm{CC} 3$, we obtain in the same way the perturbative $\mathrm{CC}(3)$ energy.

We have performed a comparative analysis of $\mathrm{CC} 3$ and CC(3) with CCSDT-1a and $\operatorname{CCSD}(\mathrm{T})$, with benchmarks against FCI and CCSDT for $\mathrm{H}_{2} \mathrm{O}, \mathrm{C}_{2}, \mathrm{CO}$, and $\mathrm{C}_{2} \mathrm{H}_{4}$. Close to equilibrium, where the reference state is dominated by a single configuration, they all give about $90 \%-95 \%$ of the effect of triples. Far from equilibrium, where static correlation is more important, they behave similarly-for one molecule some of the models work well whereas for other molecules it may be different models that work well.

In general, the iterative approximate triples methods cannot be expected to be better than the noniterative models, and vice versa. All models are constructed by perturbational arguments and in this sense the numerical tests just confirm the limitations of perturbation theory. Similarly, based on the present study, there is no reason to believe that the special treatment given to singles improves or degrades the accuracy of the total energy. It therefore appears that $\mathrm{CC} 3$ for many purposes can be viewed as a good approximation to CCSDT, with an accuracy and a robustness with respect to static correlation that is similar to that of $\operatorname{CCSD}(\mathrm{T})$. However, the advantage of CC3 is that it is well suited for calculations of time-dependent properties in contrast to $\operatorname{CCSD}(\mathrm{T})$. The response properties of the CC3 model is the subject of a subsequent publication.

\section{ACKNOWLEDGMENTS}

This work has been partly supported by the Danish Natural Research Council (Grant No. 11-0924) and the Spanish CICYT (Project No. PB94-0993).

${ }^{1}$ R. J. Bartlett, J. Phys. Chem. 93, 1697 (1989).

${ }^{2}$ J. Noga and R. J. Bartlett, J. Chem. Phys. 86, 7041 (1987) 89, 3401 (1988), and references therein.

${ }^{3}$ G. Scuseria and H. F. Schaefer, Chem. Phys. Lett. 152, 382 (1988).

${ }^{4}$ Y. S. Lee and R. J. Bartlett, J. Chem. Phys. 80, 4371 (1984).

${ }^{5}$ Y. S. Lee, S. A. Kucharski, and R. J. Bartlett, J. Chem. Phys. 81, 5906 (1984).

${ }^{6}$ M. Urban, J. Noga, S. J. Cole, and R. J. Bartlett, J. Chem. Phys. 83, 4041 (1985).

${ }^{7}$ R. J. Bartlett, H. Sekino, and G. D. Purvis, Chem. Phys. Lett. 98, 66 (1983).

${ }^{8}$ K. Raghavachari, G. W. Trucks, J. A. Pople, and M. Head-Gordon, Chem. Phys. Lett. 157, 479 (1989).

${ }^{9}$ M. J. O. Deegan and P. J. Knowles, Chem. Phys. Lett. 227, 321 (1994).

${ }^{10}$ R. J. Bartlett, J. D. Watts, S. A. Kucharski, and J. Noga, Chem. Phys. Lett. 165, 513 (1990)

${ }^{11}$ O. Christiansen, H. Koch, and P. Jørgensen, J. Chem. Phys. Nov. 1995.

${ }^{12}$ S. A. Kucharski and R. J. Bartlett, Chem. Phys. Lett. 237, 264 (1995).

${ }^{13}$ T. Helgaker and P. Jørgensen, Theor. Chim. Acta 75, 111 (1989); P. Jørgensen and T. Helgaker, J. Chem. Phys. 89, 1560 (1988); T. Helgaker and P. Jørgensen, in Methods in Computational Molecular Physics edited by S. Wilson and G. H. F. Diercksen (Plenum Press, New York, 1992), p. 353.

${ }^{14}$ J. Arponen, Ann. Phys. 151, 311 (1983).

${ }^{15}$ R. J. Bartlett, in Geometrical Derivatives of Energy Surfaces and Molecular Properties, edited by P. Jørgensen and J. Simons (Reidel, Dordrecht, the Netherlands, 1981), p. 35.

${ }^{16}$ T. Helgaker and P. Jørgensen, Adv. Quantum Chem. 19, 183 (1988).

${ }^{17}$ H. Koch, H. J. Aa. Jensen, T. Helgaker, P. Jørgensen, G. Scuseria, and H. F. Schaeffer, III, J. Chem. Phys. 92, 4924 (1990).

${ }^{18}$ H. Koch, O. Christiansen, R. Kobayashi, P. Jørgensen, and T. Helgaker, Chem. Phys. Lett. 228, 233 (1994).

${ }^{19}$ J. Olsen, P. Jørgensen, H. Koch, A. Balkova, and R. J. Bartlett, J. Chem. Phys. 104, 8007 (1996).

${ }^{20}$ ACESII an $a b$ initio program system by J. F. Stanton, J. Gauss, J. O. Watts, W. J. Lauderdale, and R. J. Bartlett.

${ }^{21}$ T. H. Dunning, Jr., J. Chem. Phys. 90, 1007 (1989). 